\title{
Rapid Urban Land Expansion in Earthquake-Prone Areas of China
}

\author{
Qingxu Huang $^{1,2} \cdot$ Shiting Meng ${ }^{1,2} \cdot$ Chunyang He ${ }^{1,2} \cdot$ Yinyin Dou $^{1,5} \cdot$ \\ Qiang Zhang ${ }^{3,4,5}$
}

Published online: 12 December 2018

(C) The Author(s) 2018

\begin{abstract}
A timely understanding of urban expansion in earthquake-prone areas is crucial for earthquake risk assessment and urban planning for earthquake mitigation. However, a comprehensive evaluation of urban expansion in earthquake-prone areas is lacking in China, especially in the context of rapid urbanization. Based on time series urban land data and seismic ground-motion parameter zonation maps, this study analyzed urban expansion in the most seismically hazardous areas (MSHAs) of China from 1992 to 2015 on the national, regional, and city scales. The results show that urban land area in the MSHAs expanded by $6767 \mathrm{~km}^{2}$ from 1992 to 2015 , with a gain of $350 \%$. Specifically, the increase in urban land area of small cities in the MSHAs of western China during this period was the fastest, 6.24 times greater than that at the national level. In terms of spatial patterns, the urban land patches in the MSHAs in 2015 were more fragmented than those in 1992
\end{abstract}

Chunyang $\mathrm{He}$

hcy@bnu.edu.cn

1 Center for Human-Environment System Sustainability (CHESS), State Key Laboratory of Earth Surface Processes and Resource Ecology (ESPRE), Beijing Normal University, Beijing 100875, China

2 School of Natural Resources, Faculty of Geographical Science, Beijing Normal University, Beijing 100875, China

3 Key Laboratory of Environmental Changes and Natural Disaster, Ministry of Education, Beijing Normal University, Beijing 100875, China

4 State Key Laboratory of Earth Surface Processes and Resource Ecology (ESPRE), Beijing Normal University, Beijing 100875, China

5 Academy of Disaster Reduction and Emergency Management, Faculty of Geographical Science, Beijing Normal University, Beijing 100875, China on all scales. The percentage of change in the number of patches and the landscape shape index of the urban land patches of small cities in the MSHAs of western China were the highest across all cities. Therefore, we believe that special attention should be paid to the cities in the MSHAs that exhibit the most rapid increases in both urban land area and fragmentation, especially the small cities in western China. It is imperative to integrate earthquake mitigation into the urban planning of these cities.

Keywords China $\cdot$ Most seismically hazardous areas · Seismic hazard exposure $\cdot$ Urbanization $\cdot$ Urban expansion · Urban sustainability

\section{Introduction}

The most seismically hazardous areas (MSHAs) represent regions where earthquakes occur frequently and are associated with severe fatalities, building damage, and economic losses (Holzer and Savage 2013; Storchak et al. 2015; NGDC-WDS 2017). Urban expansion is a process through which nonurban land is transformed into urban land (López et al. 2001; Bai et al. 2014). When urban land expanded in the MSHAs, the people, buildings, and assets exposed to seismic risk may increase. On the one hand, urban expansion can result in substantial increases in both the number and the urban land area of cities in the MSHAs (Bilham 1988). On the other hand, unregulated construction codes, and widespread weak earthquake emergency response during the rapid urbanization process will further increase seismic risk (Wu et al. 2013; Uysal et al. 2018). Therefore, a timely analysis of urban expansion in the MSHAs is of great importance for seismic-risk assessment and urban planning for earthquake mitigation. 
China is one of the countries that is most affected by earthquakes in the world. According to historical records, among all countries, China had the greatest number of earthquake fatalities during 1900-2008 (Bilham 2009). Many Chinese cities have suffered severe damages from earthquakes throughout history (Bilham 2009). For example, the death toll in the 1976 Tangshan Earthquake exceeded 240,000, and the direct economic loss was nearly RMB 13.3 billion Yuan. ${ }^{1}$ Beijing and Tianjin were also severely hit by this earthquake. The 2008 Wenchuan Earthquake caused approximately 87,000 fatalities and a direct economic loss of RMB 852.3 billion Yuan (Shi 2016).

In the past several decades, China has experienced rapid urbanization (Bai et al. 2014), with population, properties, and industries aggregating in cities at an unprecedented rate (Wieland et al. 2012). Meanwhile, the seismic risk of cities in the MSHAs has increased (Shi et al. 2002). By 2006, two-thirds of large cities in China with populations of over one million were located in the MSHAs (Xu et al. 2016b). In this context, it is very important to understand the characteristics, processes, and driving factors of urban expansion in the MSHAs of China (Wu et al. 2013).

Internationally there has been research on urban expansion in the MSHAs. For instance, Martins et al. (2012) analyzed the urban expansion of Miguel Island in Portugal from 1994 to 2005 based on a land-use and landcover change (LUCC) model, and identified a continuing urban growth towards the MSHAs. Wieland et al. (2012) used the city of Bishkek in Kyrgyzstan as an example, and assessed the urban expansion in the country's MSHAs on the neighborhood and building scales. In China, Gao (2003) qualitatively discussed changes in the seismic hazards of cities during urban expansion in multiple urban agglomerations in eastern China. Wang et al. (2007) assessed the urban expansion in Tangshan after the 1976 earthquake based on remotely sensed data and found that the urban land area increased by 135\% from 1976 to 2004. Schneider et al. (2015) investigated the urban expansion of four cities in the MSHAs of western China from 1988 to 2009. Their results indicated a $2-7 \%$ average annual growth rate in urban land area. But due to limited data availability, there is still no clear and comprehensive understanding of urban expansion in China's MSHAs.

Newly published time series urban land data of China from 1992 to 2015 (Xu et al. 2016a; Dou et al. 2017) and modified seismic ground motion parameter zonation (SGMPZ) maps (AQSIQ 2015) have provided fundamental and necessary data support for analyzing urban expansion in China's MSHAs. First, the time series urban land data can accurately and effectively reflect urban expansion in

\footnotetext{
$\overline{1}$ At the current value, USD $1=$ RMB 6.9.
}

China. These data were derived from nighttime light data, with an overall accuracy of $93 \%$ and an average Kappa value of 0.60 . This dataset has been applied to several studies of urban expansion in China. For example, based on this dataset, Du et al. (2018) explored the urban expansion in floodplains in China from 1992 to 2015. Second, the modified SGMPZ maps are more accurate than the previous maps that were generated in 2001, as they include information about recent earthquakes in China. These modified SGMPZ maps include high-magnitude potential focal regions as a key parameter (Liu 2015) and have a high level of reliability. The combination of these two datasets has made it possible to analyze urban expansion in the MSHAs of China.

The objective of this study is to analyze the characteristics and driving factors of urban expansion in the MSHAs of China. First, urban land data in China's MSHAs from 1992 to 2015 are obtained by overlapping the time series urban land data and the modified SGMPZ maps. Second, the changes in the area and spatial patterns of urban land in the MSHAs are quantified on the national, regional, and city scales. Finally, the factors that contributed to such changes are explored using socioeconomic data and statistical methods, and recommendations on urban planning for earthquake mitigation are discussed.

\section{Research Area and Data}

China $\left(73^{\circ} 40^{\prime} \mathrm{W}-135^{\circ} 2^{\prime} \mathrm{E}, 53^{\circ} 33^{\prime} \mathrm{N}-3^{\circ} 52^{\prime} \mathrm{S}\right.$, Fig. 1) is located at the junction of the Pacific seismic belt and the Mediterranean-Himalayan seismic belt (Wu et al. 2013). Earthquakes in China are characterized by their high frequency, great intensity, and wide distribution (Shi 2016). Spatially, these earthquakes are unevenly distributed, and the earthquakes that occur in western China are more frequent and of greater intensity (Xu et al. 2016b). From 1992 to 2015, urban land area in China increased from $1.22 \times 10^{4}$ to $7.29 \times 10^{4} \mathrm{~km}^{2}$, with an annual growth rate of $8 \%$ (Xu et al. 2016a). Meanwhile, the urban population in China increased from $3.22 \times 10^{8}$ to $7.71 \times 10^{8}$, by 139\% (NBSC 2016).

We analyzed urban expansion in the MSHAs of China on the national, regional, and city scales. At the regional scale, the country comprises eastern China, central China, and western China according to the Ministry of Housing and Urban-Rural Development (Fig. 1). At the city scale, we focused on counties and districts. To match these scales to those of statistical data, we adopted the method proposed by Gao et al. (2016), which merged districts under the same city jurisdiction into one single unit. After the merging process, there were 2287 county-level cities in China. We refer to these county-level urban entities as cities 


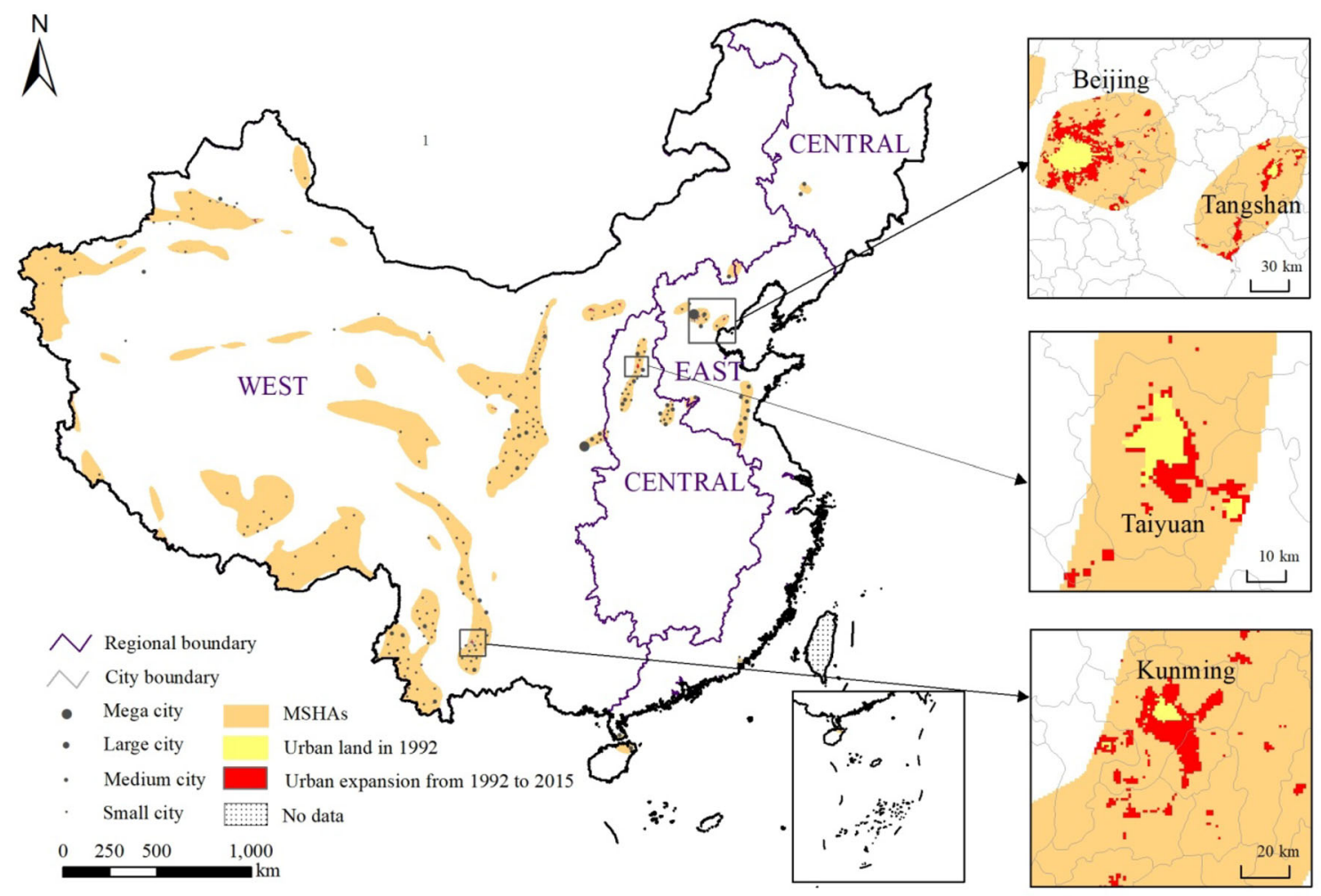

Fig. 1 Regions in China where the most seismically hazardous areas and rapid urbanization coincide. Although present in all major zones, regions of high seismic hazard and rapid urbanization are most prominent in western China. Notes: Eastern China includes eight provinces (Liaoning, Hebei, Shandong, Jiangsu, Zhejiang, Fujian, Guangdong, Hainan) and three municipalities (Beijing, Tianjin,

throughout the article. Due to the lack of statistical data, Hong Kong, Macao, and Taiwan were not included in the study.

Four types of data were used in this study. First, the time series urban land data of China from 1992 to 2015 were obtained from Xu et al. (2016a) and Dou et al. (2017). These data were based on nighttime light, vegetation index, and land surface temperature data. The spatial resolution of the data was $1 \mathrm{~km}$, with an average Kappa of 0.60, and the overall accuracy, average quantity of disagreement, and average allocation of disagreement were $93 \%, 1.5 \%$, and $6 \%$, respectively. The reliability of the urban land data has been tested by urban land data based on the Landsat TM/ ETM + images (Xu et al. 2016a). Specifically, five capital cities (Beijing, Nanjing, Kunming, Zhengzhou, Nanchang) with different levels of urbanization were selected to assess the spatial accuracy of the data. Among the five cities, Beijing and Kunming are located in the MSHAs. The Kappa value was 0.42 and 0.67 in the two cities. The average overall accuracy, average quantity of disagreement, and average allocation of disagreement were above $84 \%, 3.7 \%$, and $12 \%$, respectively.
Shanghai); central China includes eight provinces (Heilongjiang, Jilin, Shanxi, Henan, Anhui, Hubei, Hunan, Jiangxi); western China includes one municipality (Chongqing), five autonomous regions (Inner Mongolia, Xinjiang, Ningxia, Tibet, Guangxi), and six provinces (Gansu, Qinghai, Shaanxi, Sichuan, Guizhou, Yunnan). MSHAs $=$ most seismically hazardous areas

Second, the peak ground acceleration (PGA) data were derived from the modified SGMPZ maps (AQSIQ 2015). These PGA values have a $10 \%$ probability of being exceeded in a span of 50 years. Third, GDP of primary industry, GDP of secondary industry, GDP of tertiary industry, and total investment in the fixed assets of each province were obtained from the China Statistical Yearbook (NBSC 1993, 2016). The total population and urban population data were obtained from the 2010 population census of China (NBSC-PCO 2012). In addition, the 1:4,000,000 urban administrative boundary data were collected from the National Geomatics Center of China. ${ }^{2}$

\section{Method}

The MSHAs in China were defined based on the classification in the SGMPZ maps of China. We selected the areas where the PGA value was equal to or greater than $0.2 \mathrm{~g}$ (in which $g$ is the gravitational acceleration) as the MSHAs, following the method used by Holzer and Savage (2013).

\footnotetext{
${ }^{2}$ http://www.ngcc.cn/article/en/.
} 
Under this definition, the MSHAs are equivalent to areas with seismic impacts of greater than VIII according to the national standard GB-T 17742-2008 (AQSIQ and SAC 2008).

\subsection{Identifying the Cities in the Most Seismically Hazardous Areas}

The cities in the MSHAs were determined by overlapping the MSHA layer and the urban administrative boundary layer. According to the method employed by Potere et al. (2009) and He et al. (2016), the cities in the MSHAs refer to those cities in which more than $50 \%$ of the total area within their administrative boundaries was characterized as being a MSHA. This identification revealed that there were 239 cities in China's MSHAs. Furthermore, we classified these cities by their population size in 2010 according to Bai et al. (2014). There were two mega cities (with a total population of greater than or equal to 5 million), 16 large cities (with a total population between 1 million and 5 million), 43 medium cities (with a total population between 0.5 million and 1 million), and 178 small cities (with a total population of less than or equal to 0.5 million). These are displayed in Fig. 1.

\subsection{Analyzing Changes in the Urban Land Area in the Most Seismically Hazardous Areas}

Changes in the urban land area in the MSHAs in China from 1992 to 2015 were analyzed on the national, regional, and city scales. These changes can be calculated as follows,

$\mathrm{Urb}_{\Delta k}=\sum_{i=1}^{n} \operatorname{urb}_{(2015, i)}-\sum_{i=1}^{n} \operatorname{urb}_{(1992, i)}$

where $\operatorname{Urb}_{(\Delta k)}$ is the change in the urban land area in the MSHAs from 1992 to 2015; and $\operatorname{urb}_{(2015, i)}$ and $\operatorname{urb}_{(1992, i)}$ are the urban land areas of pixel $i$ in the MSHAs in 2015 and 1992, respectively.

\subsection{Quantifying Changes in the Spatial Patterns of Urban Land in the Most Seismically Hazardous Areas}

We chose three landscape metrics to measure the changes in the spatial patterns of urban land in the MSHAs, by adopting the indicators of $\mathrm{Wu}$ et al. (2011) and Liu et al. (2016). The changes in the spatial patterns of urban land in the MSHAs can be calculated as follows,

$\operatorname{Inx}_{(j, \Delta k)}=\operatorname{Inx}_{(j, 2015)}-\operatorname{Inx}_{(j, 1992)}$

where $\operatorname{Inx}(j, \Delta k)$ is the change in the landscape metric $j$ of urban land in the MSHAs from 1992 to 2015 ; and $\operatorname{Inx}(j, 2015)$ and $\operatorname{Inx}(j, 1992)$ are the values of the landscape metric $j$ of urban land in the MSHAs in 2015 and 1992, respectively.

The three landscape metrics were the number of patches (NP), patch density (PD), and landscape shape index (LSI), all of which were calculated by Fragstats 4.2 (McGarigal 2014). The equations used for the three landscape metrics are as follows,

$\mathrm{NP}=N$

where $N$ is the number of patches. NP indicates the amount of urban land patches in the MSHAs. The greater the NP is, the more the urban land patches in the MSHAs there are.

$\mathrm{PD}=\frac{N}{\mathrm{~A}}$

where $A$ is the total area of land $\left(\mathrm{km}^{2}\right)$. PD indicates the level of fragmentation of urban land patches in the MSHAs. The fragmentation of the urban land patches in the MSHAs increases with PD.

$\mathrm{LSI}=\frac{0.25 E}{\sqrt{A}}$

where $E$ is the length of the patch boundaries (m), and 0.25 is the square correction constant. LSI represents the shape regularity of the urban land patches in the MSHAs. The irregularity of the urban land patches in the MSHAs increases with LSI.

\subsection{Examining Urban Expansion Modes in the Most Seismically Hazardous Areas}

Urban expansion modes refer to the spatial relationship between existing urban land and new urban land during the urban growth process. We used the landscape expansion index (LEI) proposed by Liu et al. (2010) to examine the urban expansion modes in the MSHAs from 1992 to 2015. The LEI can be calculated by the following formula,

LEI $=\frac{A_{0}}{A_{0}+A_{V}} \times 100$

where $A_{0}$ is the area of overlap between a buffer zone of the new urban land patch and the existing urban land, and $A_{\mathrm{V}}$ is the area of overlap between the same buffer zone and the existing nonurban land. Following previous studies (Liu et al. 2010; Xu et al. 2016a), the radius of the buffer zone was set to $200 \mathrm{~m}$, and the LEI of the new urban land patch was calculated annually.

Based on the LEI, the urban expansion modes can be divided into three types: infill, leapfrog, and edge-expansion. If the LEI is between 50 and 100, then the urban expansion is defined as the infill mode, which means that the gaps within the existing urban land area are filled by new urban land patches. The leapfrog mode refers to the 
situation in which the LEI is equal to 0 and the new urban land patches are isolated from existing ones. Otherwise, the urban expansion falls into the edge-expansion mode, which represents that new urban land patches expand around the edge of the existing urban land.

\section{Results}

In this section, we present the characteristics of urban expansion in the MSHAs in three aspects-the changes in land area, landscape metrics of urban land patches and urban expansion modes in the MSHAs of China during 1992-2015.

\subsection{Changes in Urban Land Area in the Most Seismically Hazardous Areas of China from 1992 to 2015}

Urban land area in the MSHAs of China increased steadily from 1992 to 2015 (Fig. 2a). At the national level, the urban land area in the MSHAs increased from $1935 \mathrm{~km}^{2}$ in 1992 to $8702 \mathrm{~km}^{2}$ in 2015 (Fig. 2b), an expansion of almost 350\% (Table 1).

At the regional level, urban land area in the MSHAs in western China experienced the most rapid growth (Fig. 2c). During 1992-2015, urban land area in the MSHAs in western China increased from 781 to $4884 \mathrm{~km}^{2}$, a gain of $525 \%$ (Table 1). This expansion was 1.5 times greater than that of urban land area in the MSHAs at the national level and 2.2 and 2.5 times greater than those of eastern China and central China, respectively.

Among different types of cities in China, urban land area of small cities in the MSHAs in western China featured the fastest growth (Fig. 2d). The urban land area of those cities increased by $2181 \%$, from $72 \mathrm{~km}^{2}$ in 1992 to $1642 \mathrm{~km}^{2}$ in 2015 (Table 1). Specifically, the percentage of increase of the urban land area of small cities in the MSHAs of western China was 6.2 times greater than that at the national level, and slightly more than double that of other cities in the MSHAs.

\subsection{Changes in the Spatial Patterns of Cities in the Most Seismically Hazardous Areas from 1992 to 2015}

From 1992 to 2015, the urban land patches in the MSHAs of China became more fragmented. At the national level, all three landscape metrics exhibited substantial increases, and the change of NP, PD, and LSI were $823 \%, 107 \%$, and $168 \%$, respectively (Table 2 ).

At the regional level, the degree of fragmentation of the urban land patches in the MSHAs of western China was the highest among the three regions. The NP of the urban land patches in the MSHAs of western China increased by $1189 \%$ (Table 2), which was 1.4 times greater than the national average and 2.09 and 0.83 times greater than that of eastern China and central China, respectively. Meanwhile, the LSI of the urban land patches increased by $183 \%$ (Table 2) and was $15.5 \%, 48 \%$, and $64 \%$ higher than those at the national level and of eastern and central China, respectively.

Similarly, at the city scale, the urban land patches of small cities in the MSHAs of western China experienced the most rapid process of fragmentation. The percentage of increase of the NP and LSI of the urban land patches in small cities in the MSHAs of western China ranked first among all the cities in the MSHAs. Specifically, the NP and LSI increased by $2822 \%$ and $371 \%$ (Table 2) or were 3.43 and 2.21 times greater than those at the national level, respectively. In other words, the percentage of increase of the NP and LSI of the urban land patches of the small cities in the MSHAs of western China were more than 1.71 and 1.08 times greater than those of the other cities in the MSHAs, respectively.

\subsection{Urban Expansion Modes in the Most Seismically Hazardous Areas from 1992 to 2015}

During 1992-2015, edge expansion was the dominant urban expansion mode of the cities in the MSHAs of China (Table 3). The total area of the edge-expansion mode in the cities in the MSHAs was $4024 \mathrm{~km}^{2}$, accounting for nearly $60 \%$ of the total urban expansion area in the MSHAs. In addition, the areas of the leapfrog mode and infilling mode were $1286 \mathrm{~km}^{2}$ and $1425 \mathrm{~km}^{2}$, accounting for $19 \%$ and $21 \%$ of the total urban expansion area in the cities in the MSHAs, respectively (Table 3).

At the regional scale, the dominant urban expansion mode in the MSHAs was also edge expansion, which accounted for $58-61 \%$ of the total urban expansion area among the three regions in the MSHAs (Table 3). It is worth noting that the percentage of the leapfrog mode area of cities in the MSHAs of western China was the highest among those of the three regions. The area of the leapfrog mode accounted for almost $23 \%$ of the total urban expansion area in the MSHAs of western China, which was nearly $1.2,2$, and 1 times greater than its proportions at the national level and of eastern and central China, respectively.

For all cities in the MSHAs, the area of the edge-expansion mode accounted for 54-69\% of the total urban expansion area in each city in the MSHAs (Table 3). The small cities in the MSHAs of western China had the highest percentage of leapfrog urban area, which is consistent with previous findings that the small cities in the MSHAs of 
Fig. 2 Changes in urban land area in the most seismically hazardous areas of China, 1992-2015. a Spatial dynamics of urban land area in the MSHAs; b changes in total urban land area in the MSHAs; c percentage of increase of urban land area in the MSHAs at the national and regional levels; $\mathbf{d}$ percentage of increase of urban land area in the MSHAs at the city level
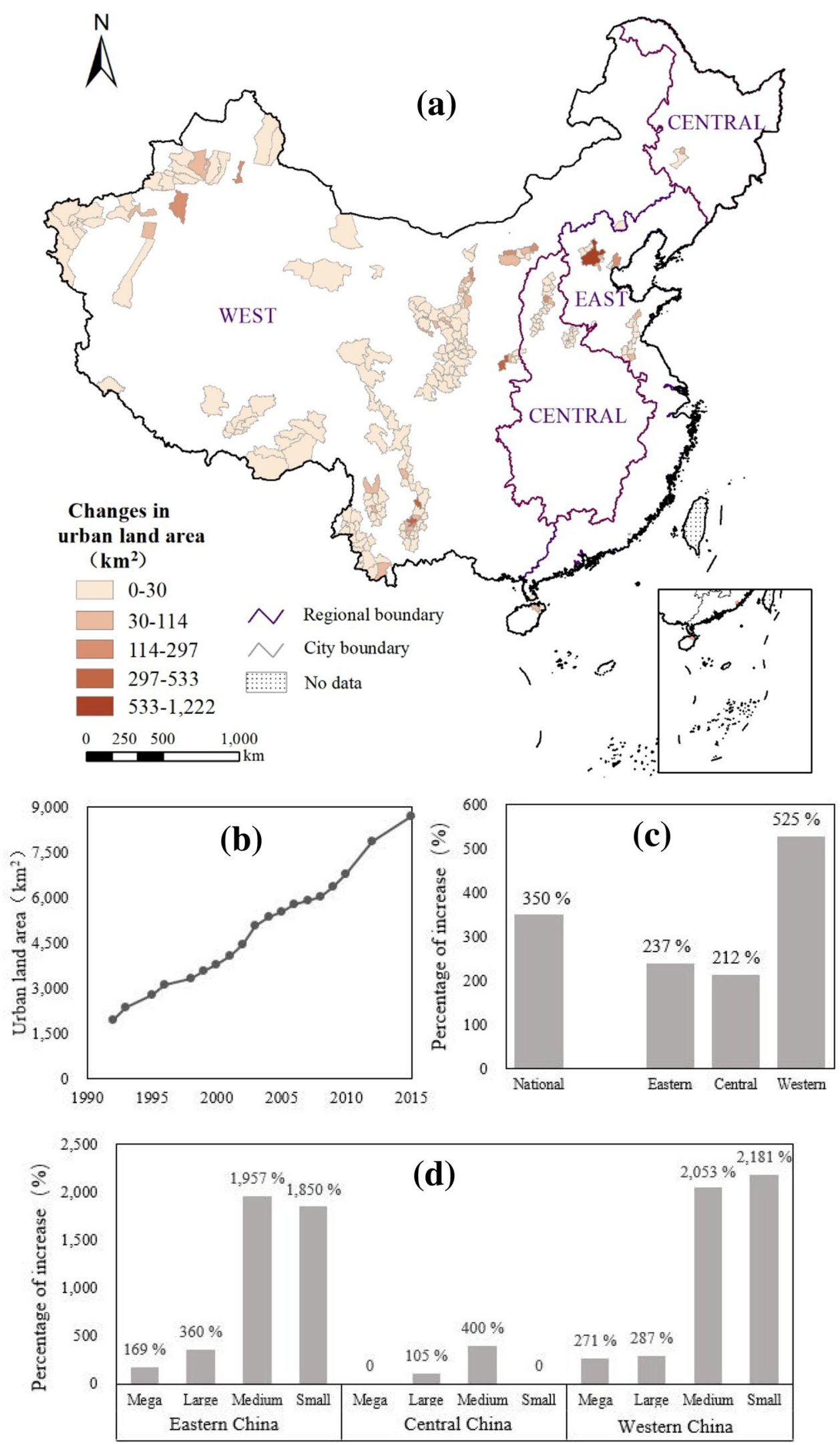
Table 1 Changes in urban land areas in the most seismically hazardous areas of China, 1992-2015

\begin{tabular}{|c|c|c|c|c|}
\hline Scale & $1992\left(\mathrm{~km}^{2}\right)$ & $2015\left(\mathrm{~km}^{2}\right)$ & $1992-2015\left(\mathrm{~km}^{2}\right)$ & Change rate $(\%)$ \\
\hline \multicolumn{5}{|l|}{ China } \\
\hline Total cities & 1935 & 8688 & 6767 & 350 \\
\hline Mega cities & 922 & 2677 & 1755 & 190 \\
\hline Large cities & 839 & 2925 & 2086 & 249 \\
\hline Medium cities & 96 & 1194 & 1098 & 1144 \\
\hline Small cities & 78 & 1892 & 1828 & 2326 \\
\hline \multicolumn{5}{|l|}{ Eastern } \\
\hline Total cities & 873 & 2941 & 2068 & 237 \\
\hline Mega cities & 725 & 1947 & 1222 & 169 \\
\hline Large cities & 128 & 589 & 461 & 360 \\
\hline Medium cities & 14 & 288 & 274 & 1957 \\
\hline Small cities & 6 & 117 & 111 & 1850 \\
\hline \multicolumn{5}{|l|}{ Central } \\
\hline Total cities & 281 & 877 & 596 & 212 \\
\hline Mega cities & - & - & - & - \\
\hline Large cities & 229 & 470 & 241 & 105 \\
\hline Medium cities & 52 & 260 & 208 & 400 \\
\hline Small cities & 0 & 147 & 147 & - \\
\hline \multicolumn{5}{|l|}{ Western } \\
\hline Total cities & 781 & 4884 & 4103 & 525 \\
\hline Mega cities & 197 & 730 & 533 & 271 \\
\hline Large cities & 482 & 1866 & 1384 & 287 \\
\hline Medium cities & 30 & 646 & 616 & 2053 \\
\hline Small cities & 72 & 1642 & 1570 & 2181 \\
\hline
\end{tabular}

The bold text is used to highlight the results from focal area

western China experienced rapid increases in both urban land area and degree of fragmentation. The area of leapfrog-mode expansion accounted for more than $36 \%$ of the total urban expansion area of small cities in the MSHAs of western China. This proportion was almost two times or more than 1.2 times greater than the proportion at the national level and those of other cities in the MSHAs, respectively.

\section{Discussion}

We discuss the reliability of our results and the implication of our findings in this section. In addition, the highlights and future perspectives of this research are also discussed.

\subsection{Reliability of the Results Based on the Most Seismically Hazardous Areas}

Different sources of PGA data may influence the distribution of the MSHAs and may thus further affect results. To validate the reliability of our major findings, we compared the outcomes obtained based on the SGMPZ maps used in this study and another PGA dataset obtained from the Global Seismic Hazard Assessment Program (GSHAP) (Giardini et al. 1999) at the city level. The results indicate that using the GSHAP PGA dataset would not alter our major findings. First, the changes in the urban land area in the MSHAs of China based on the two datasets showed a significantly positive correlation $(r=0.76, p<0.05$, Table 4). This suggests that the urban expansion process in the MSHAs derived from the two datasets was consistent. Second, the changes in the spatial patterns of urban land in the MSHAs based on the two datasets were also significantly correlated. The changes in the NP of urban land patches in the MSHAs were highly and significantly correlated at the city level $(r=0.99, p<0.01)$, and the changes in the LSI of urban land patches in the MSHAs showed a similar trend $(r=0.96, p<0.05$, Table 4$)$. 
Table 2 Changes in the NP, PD, and LSI of urban land patches in the most seismically hazardous areas of China, 1992-2015

\begin{tabular}{|c|c|c|c|c|c|c|c|c|c|}
\hline \multirow[t]{2}{*}{ Scale } & \multicolumn{3}{|c|}{ NP (number) } & \multicolumn{3}{|c|}{$\mathrm{PD}$ (number/km²) } & \multicolumn{3}{|c|}{ LSI (no unit) } \\
\hline & 1992 & 2015 & Change (\%) & 1992 & 2015 & Change $(\%)$ & 1992 & 2015 & Change $(\%)$ \\
\hline \multicolumn{10}{|l|}{ China } \\
\hline Total cities & 56 & 517 & 823 & 0.029 & 0.060 & 106 & 8.16 & 21.86 & 168 \\
\hline Mega cities & 13 & 41 & 215 & 0.01 & 0.02 & 8.6 & 3.69 & 6.63 & 80 \\
\hline Large cities & 23 & 94 & 308 & 0.03 & 0.03 & 17 & 6.10 & 10.09 & 65 \\
\hline Medium cities & 10 & 102 & 920 & 0.10 & 0.09 & -18 & 3.40 & 11.04 & 225 \\
\hline Small cities & 10 & 280 & 2700 & 0.01 & 0.15 & 1107 & 1.17 & 17.75 & 1421 \\
\hline \multicolumn{10}{|l|}{ Eastern } \\
\hline Total cities & 20 & 97 & 385 & 0.023 & 0.033 & 44 & 4.18 & 9.85 & 135 \\
\hline Mega cities & 9 & 33 & 267 & 0.012 & 0.018 & 45 & 3.00 & 6.00 & 100 \\
\hline Large cities & 6 & 29 & 383 & 0.047 & 0.059 & 27 & 2.83 & 5.92 & 109 \\
\hline Medium cities & 3 & 25 & 733 & 0.214 & 0.094 & -56 & 1.75 & 5.50 & 214 \\
\hline Small cities & 2 & 10 & 400 & 0.333 & 0.085 & -74 & 2.00 & 3.55 & 77 \\
\hline \multicolumn{10}{|l|}{ Central } \\
\hline Total cities & 8 & 60 & 650 & 0.028 & 0.068 & 140 & 3.21 & 7.03 & 119 \\
\hline Mega cities & - & - & - & - & - & - & - & - & - \\
\hline Large cities & 3 & 10 & 267 & 0.013 & 0.023 & 79 & 2.39 & 3.23 & 35 \\
\hline Medium cities & 5 & 17 & 240 & 0.096 & 0.081 & -16 & 2.33 & 4.73 & 103 \\
\hline Small cities & 0 & 33 & - & 0.000 & 0.224 & - & 0.00 & 6.04 & - \\
\hline \multicolumn{10}{|l|}{ Western } \\
\hline Total cities & 28 & 361 & 1189 & 0.036 & 0.074 & 106 & 6.54 & 18.52 & 183 \\
\hline Mega cities & 4 & 11 & 175 & 0.020 & 0.015 & -26 & 2.17 & 3.11 & 43 \\
\hline Large cities & 13 & 52 & 300 & 0.029 & 0.028 & -4 & 5.05 & 7.76 & 54 \\
\hline Medium cities & 2 & 35 & 1650 & 0.067 & 0.091 & 37 & 1.73 & 7.65 & 342 \\
\hline Small cities & 9 & 263 & 2822 & 0.125 & 0.160 & 28 & 3.56 & 16.76 & 371 \\
\hline
\end{tabular}

The bold text is used to highlight the results from focal area

\subsection{Urban Population Growth is an Important Factor During Urban Expansion in the Most Seismically Hazardous Areas}

Previous studies found that socioeconomic development can accelerate urban expansion in the MSHAs (Seto et al. 2011). Following the method proposed by Tao et al. (2015) and Chatterje et al. (2000), we adopted the Pearson correlation analysis and general linear regression model (GLM) to explore the relationship between socioeconomic development and the increase in the urban land area of the MSHAs. Three social indicators (total population, urban population, and urbanization rate) and five economic indicators (GDP, GDP of primary industry, GDP of secondary industry, GDP of tertiary industry, and total investment in fixed assets) were selected as such data were available.

We found that the increase in urban land area in the MSHAs was positively correlated with the growth of multiple socioeconomic indicators, and these correlations passed the significance test at the 0.01 level (Fig. 3). Among these factors, the increase in the total investment in fixed assets had the highest correlation with the increase in urban land area in the MSHAs $(r=0.975$, Table 5), followed by the increases in GDP $(r=0.948)$ and urban population $(r=0.945)$. The GLM analysis indicates that the increase in urban population was the main factor driving urban expansion in the MSHAs. The increase in urban population accounted for approximately $96 \%$ of the total variations in the changes of urban land area in the MSHAs (Table 5). This finding was consistent with that of Schneider et al. (2015), who found that urban population growth accelerated rapid urban expansion in western China during 1988-2010.

In addition, national policies also played an important role in encouraging urban expansion in the MSHAs. Since 1989, China's central government has made efforts to develop small and medium cities (City Planning Law of China in $1989^{3}$ ), as

\footnotetext{
${ }^{3}$ http://www.npc.gov.cn/englishnpc/news/index.htm.
} 
Table 3 Urban expansion modes of the cities in the most seismically hazardous areas in China, 1992-2015

\begin{tabular}{|c|c|c|c|c|c|c|}
\hline \multirow[t]{2}{*}{ Scale } & \multicolumn{2}{|l|}{ Leapfrog } & \multicolumn{2}{|l|}{ Infill } & \multicolumn{2}{|c|}{ Edge expansion } \\
\hline & Area $\left(\mathrm{km}^{2}\right)$ & Proportion (\%) & Area $\left(\mathrm{km}^{2}\right)$ & Proportion (\%) & Area $\left(\mathrm{km}^{2}\right)$ & Proportion (\%) \\
\hline \multicolumn{7}{|l|}{ China } \\
\hline Total cities & 1286 & 19.1 & 1425 & 21.2 & 4024 & 59.8 \\
\hline Mega cities & 120 & 6.9 & 604 & 34.7 & 1017 & 58.4 \\
\hline Large cities & 229 & 11.0 & 564 & 27.1 & 1290 & 61.9 \\
\hline Medium cities & 277 & 25.3 & 124 & 11.3 & 693 & 63.4 \\
\hline Small cities & 660 & 36.3 & 133 & 7.3 & 1024 & 56.4 \\
\hline \multicolumn{7}{|l|}{ Eastern } \\
\hline Total cities & 247 & 11.9 & 554 & 26.8 & 1269 & 61.3 \\
\hline Mega cities & 94 & 7.7 & 398 & 32.5 & 733 & 59.8 \\
\hline Large cities & 69 & 15.0 & 108 & 23.5 & 283 & 61.5 \\
\hline Medium cities & 56 & 20.5 & 28 & 10.3 & 189 & 69.2 \\
\hline Small cities & 28 & 25.0 & 20 & 17.9 & 64 & 57.1 \\
\hline \multicolumn{7}{|l|}{ Central } \\
\hline Total cities & 129 & 21.8 & 108 & 18.2 & 355 & 60.0 \\
\hline Mega cities & - & - & - & - & - & - \\
\hline Large cities & 12 & 4.8 & 78 & 31.0 & 162 & 64.3 \\
\hline Medium cities & 38 & 18.7 & 26 & 12.8 & 139 & 68.5 \\
\hline Small cities & 79 & 27.7 & 4 & 2.9 & 54 & 69.4 \\
\hline \multicolumn{7}{|l|}{ Western } \\
\hline Total cities & 921 & 22.9 & 763 & 19.0 & 2333 & 58.1 \\
\hline Mega cities & 26 & 5.2 & 206 & 40.9 & 272 & 54.0 \\
\hline Large cities & 148 & 10.9 & 378 & 27.8 & 836 & 61.4 \\
\hline Medium cities & 185 & 30.4 & 70 & 11.5 & 353 & 58.1 \\
\hline Small cities & 562 & 36.4 & 109 & 7.1 & 872 & 56.5 \\
\hline
\end{tabular}

The bold text is used to highlight the results from focal area

Table 4 Comparison of the results derived from different PGA datasets

\begin{tabular}{llc}
\hline Indicators of the cities in the MSHAs & \multicolumn{2}{c}{ (Number of samples: 12) ${ }^{\mathrm{a}}$} \\
\cline { 2 - 3 } & $r$ & $P$ \\
\hline Urban land area & 0.76 & $<0.05$ \\
NP & 0.99 & $<0.05$ \\
PD & 0.03 & $>0.05$ \\
LSI & 0.96 & $<0.05$ \\
\hline
\end{tabular}

${ }^{\mathrm{a}}$ The numbers of cities in the MSHAs were different between the two datasets. Therefore, we conducted the correlation analysis using the average values of the four indicators among four types of cities (that is, mega, large, medium, and small cities) in eastern, central, and western China's MSHA, so the number of samples was 12

well as satellite towns near large cities (China's Agenda 21 in $1994^{4}$ ) for a more coordinated development of cities of different sizes (Ma et al. 2016). Such resolutions were also written in China's Five-Year Plan in four consecutive periods

\footnotetext{
${ }^{4}$ http://www.acca21.org.cn/.
}

from 2001 to 2020. Hence, the rapid increase in urban population, especially for those small cities in the MSHAs, may have a strong relationship with such policies. Besides, the West Region Development Strategy (or the "Go West" Program ${ }^{5}$ ) that was initiated in 2000 greatly boosted the urbanization process in western China (Liu et al. 2005; Schneider et al. 2005). The "Belt and Road" initiative ${ }^{6}$ that began in 2015 may also accelerate urban expansion in the MSHAs in western China. In the future, along with China's urbanization, urban land area in the MSHAs in western China may continue to increase the momentum of rapid urbanization (Schneider et al. 2015; Gao et al. 2016).

\footnotetext{
5 http://en.ndrc.gov.cn/mfod/200812/t20081217_252127.html.

${ }^{6}$ http://en.ndrc.gov.ex2.ipv6.ndrc.gov.cn/newsrelease/201503/ t20150330_669367.html.
} 
Fig. 3 Relationship between the increases in urban land area in the most seismically hazardous areas and the changes in socioeconomic factors, 1992-2015
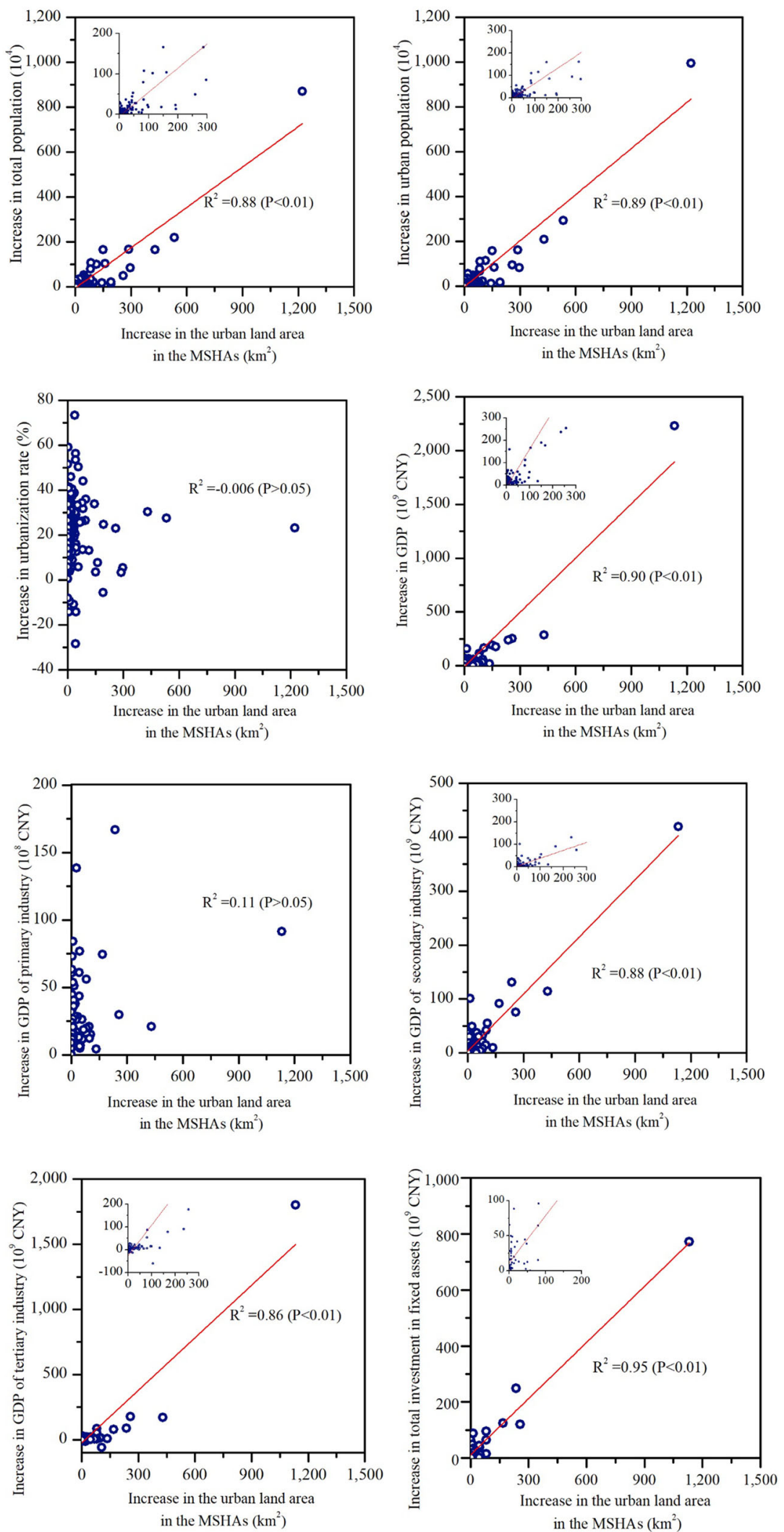
Table 5 Relationships between changes in urban land areas in the most seismically hazardous areas and changes in socioeconomic factors, 1992-2015

\begin{tabular}{lcl}
\hline Factors & $r$ & $P$ \\
\hline Pearson correlation analysis & & \\
Total population & 0.94 & $<0.01$ \\
Urban population & 0.95 & $<0.01$ \\
Urbanization rate & 0.05 & $>0.05$ \\
GDP & 0.95 & $<0.01$ \\
GDP of primary industry & 0.34 & $>0.05$ \\
GDP of secondary industry & 0.94 & $<0.01$ \\
GDP of tertiary industry & 0.93 & $<0.01$ \\
Total investment in fixed assets & 0.97 & $<0.01$ \\
\hline Factors & MS & $\mathrm{SS}, \%$ \\
\hline General linear model & & \\
Total population & 0.07 & 0.05 \\
Urban population & 147.94 & $95.74^{\mathrm{a}}$ \\
GDP & 0.12 & 0.08 \\
GDP of secondary industry & 2.42 & $1.57^{\mathrm{a}}$ \\
GDP of tertiary industry & 0.25 & 0.16 \\
Total investment in fixed assets & 0.23 & 0.15 \\
Residues & 0.17 & 2.27 \\
\end{tabular}

$M S$ is the mean square, and $\mathrm{SS}$ is the proportion of the variance explained by the variables

${ }^{\mathrm{a}} p<0.01$

\subsection{More Attention Should be Paid to Small Cities in the Most Seismically Hazardous Areas in Western China}

The results reveal the fact that excessive urban expansion in the MSHAs of China is taking place both quantitatively and spatially. This is especially the case for cities in western China. To achieve the goal of attracting investment and increasing fiscal revenue, local governments were encouraged to build high-tech development zones and industrial parks in western China. Such support triggered the construction boom (Gao et al. 2016), and urban land patches in the MSHAs multiplied and became more fragmented.

Small cities in the MSHAs of western China face more threats due to several reasons. First, the urbanization of small cities in western China began late but has undergone rapid acceleration in recent years (Gao et al. 2016), yet the regulations on earthquake-resistant construction of critical infrastructure is insufficient (Spence 2007). In the 2008 Wenchuan Earthquake, more than $80 \%$ of the collapsed buildings were built before the 1990s and these unreinforced buildings led to higher deaths and injuries (Wang

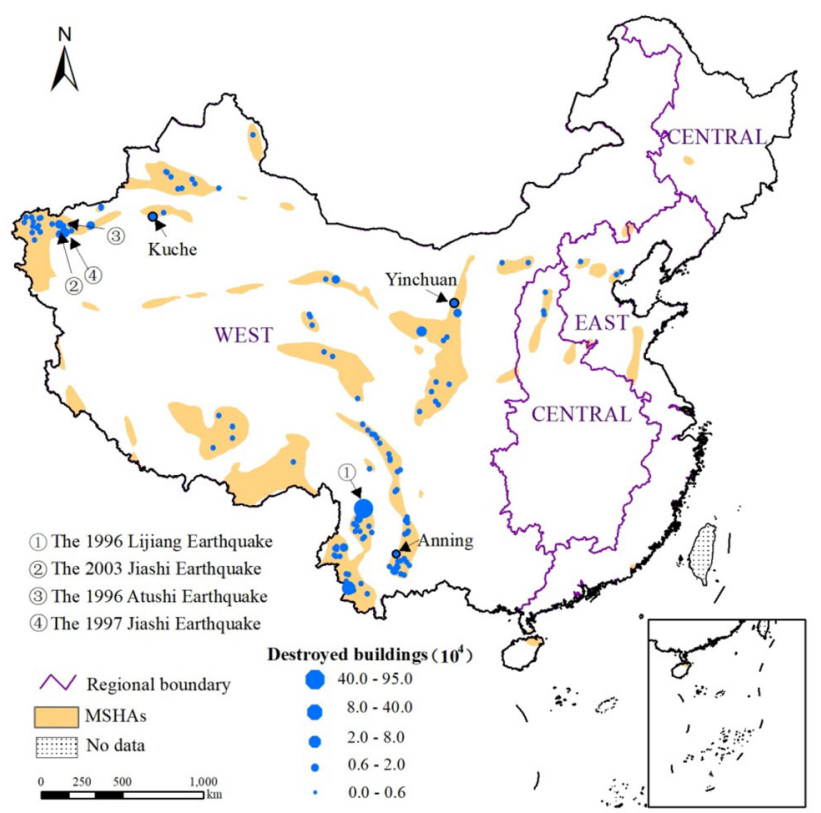

Fig. 4 The numbers and spatial distribution of buildings collapsed by earthquakes in the most seismically hazardous areas, 1900-2015. MSHAs $=$ most seismically hazardous area. Source NGDC-WDS (2017)

2008). Meanwhile, emergency response has not been adequate in small cities (MOHURD 2017). Second, small cities in the MSHAs of western China have suffered the most building damage due to earthquakes compared to other cities. Such losses are mainly attributed to the higher frequency and greater intensity of earthquakes in western China (Xu et al. 2016b) and weak seismic capacities of buildings in small cities (Sun and Zhang 2018). According to the historical data from 1900 to 2015 obtained from the National Centers for Environmental Information (NGDCWDS 2017), ${ }^{7}$ the collapsed buildings due to earthquakes were mainly located in western China (Fig. 4). From 1900 to 2015 , the number of buildings collapsed by earthquakes reached 1.72 million in western China, which accounted for more than $99 \%$ of all the buildings collapsed by earthquakes in the entire country. Small cities in the MSHAs of western China witnessed a total loss of 1.68 million collapsed buildings, which represented $98 \%$ of the total buildings collapsed by earthquakes in the MSHAs of western China. During 1992-2015, four of the five earthquakes with over 10 thousand collapsed buildings occurred in small cities in the MSHAs of western China (Fig. 4).

In the context of the steady increase of urban land in the MSHAs, special attention should be paid to the small cities in the MSHAs of western China, which feature high growth rates in their urban land area and an extreme degree of fragmentation. For example, the percentage of increase in

\footnotetext{
${ }^{7}$ https://www.ngdc.noaa.gov/hazard/earthqk.shtml.
} 
urban land areas of Anning in Yunnan Province and Kuche in Xinjiang Autonomous Region have both exceeded $900 \%$. To ensure a safe and sustainable city development, first, construction should be largely restricted in the MSHAs through urban planning. For instance, replacing high-density buildings with a green-belt park along an earthquake fault zone within a city is a feasible option, and the park can also serve as an emergency shelter. Currently in western China, Yinchuan City of Ningxia Autonomous Region is a pilot city of such policy and it is promising for other cities in the MSHAs to follow the same pattern. Second, for cities that are already located in the MSHAs, efforts under the "prevention-resistance-shelter-rescue" framework (MOHURD 2007) should be strengthened. We recommend that the detection on active faults in urban areas should be further extended from large cities to those small cities in western China for a more comprehensive understanding of potential seismic risk. Meanwhile, it is crucial to create a unified standard for earthquake resistance and prevention and to implement necessary policies to guarantee the execution of the standard (Zhang et al. 2018). Third, new technologies should be adopted to enhance seismic-risk management. For example, high-resolution optical imagery together with geological methods can be adopted for cities in the MSHAs to identify and visualize the three-dimensional human-made constructions (Ehrlich and Tenerelli 2013), and to optimize the location of earthquake emergency facilities (Jiang et al. 2018).

\subsection{Future Perspectives on Coping with Urban Expansion in Seismically High-Risk Areas}

Our research in the MSHAs of China was only an example of what has happened, is happening, or will happen across the world. Recently, many countries also experienced a similar process of urban expansion into earthquake-prone areas in the context of rapid urbanization. For example, Ehrlich et al. (2018) found that the built-up area that was exposed to earthquakes in the world increased by $145 \%$ from 1975 to 2015. Dou et al. (2018) observed that rapid urbanization contributed in large part to the growth of population in Asia's MSHAs. On that basis, it is imperative to understand urban expansion in the MSHAs and take immediate actions to mitigate the corresponding risk in a global perspective.

Compared with relevant existing research, our study featured the following three aspects. First, we performed a multiscale analysis of urban expansion process in China to identify the focal area, instead of just focusing on an individual city in the MSHAs (Wang et al. 2007). Second, we analyzed the dynamics of the spatial patterns of urban land in the MSHAs, which has not been captured by previous studies. Third, we provided an alternative to examining the exposure of urban land to seismic risk in earthquake-prone areas, apart from the exposure of population (He et al. 2016) and assets (Wu et al. 2017) to seismic risk.

This study has limitations in the following three aspects. First, the resolution of urban land data was limited. At the city level, the $1 \mathrm{~km}$ time series urban land data could not provide detailed information such as building composition within a city. Also, limited resolution may influence the accuracy of landscape metrics. Second, the factors of spatiotemporal change that drive rapid urbanization of land in China's MSHAs from 1992 to 2015 still needs to be investigated. Third, the issue of vacant housing that comes with migrant workers was not considered in this study. Since the urban land data analyzed in this study were extracted using the annual composites of the nighttime stable light data, vegetation index, and land surface temperature data, short-term nighttime light events, such as the lighting of houses occupied by migrant workers during the Spring Festival, could not be captured. Therefore, the issue of migrant workers and vacant housing may affect the accuracy of the urban land data to some extent.

In the future, for the cities in the MSHAs that require greater attention, high-resolution remote-sensing data can be used to extract building information. Further analysis on the exposure of buildings in cities in the MSHAs and the vulnerability of these cities should be conducted (Ehrlich and Tenerelli 2013) to lay the foundation for urban planning for earthquake mitigation. Moreover, Granger causality analysis (Granger 1969) and the spatial autocorrelation model ( $\mathrm{Li}$ et al. 2018). can be adopted to explore the driving forces of changes in urban land use in the MSHAs from both socioeconomic and biophysical perspectives. For the ignored vacant houses temporarily occupied by migrants, monthly composites of nighttime light data can be used to improve the accuracy of the urban land extraction (Chen et al. 2015).

\section{Conclusion}

Urban land expanded rapidly in China's MSHAs. From 1992 to 2015, urban land areas in the MSHAs in China increased by $6767 \mathrm{~km}^{2}$, which represents an increase of $350 \%$. Spatially, urban land patches in the MSHAs were more fragmented over time. The percentage of increase in the NP, PD, and LSI all exceeded $100 \%$ at the national level. Behind the changes, urban population growth played an important role in accelerating urban expansion in the MSHAs. The increase in urban population was highly correlated with an increase in the urban land area of the MSHAs $(r=0.945, \quad p<0.01)$ and accounted for 
approximately $96 \%$ of the total variations in the changes of the urban land area in the MSHAs.

The small cities in the MSHAs of western China exhibited the most rapid increases in both urban land area and patch fragmentation. The urban land area in the MSHAs in western China had increased by $2181 \%, 6.2$ times greater than comparable increase at the national scale. Meanwhile, the percentage of increases in the NP, LSI of the urban land patches, and the percentage of the leapfrog urban expansion in the MSHAs in western China were $2822 \%, 371 \%$, and $36 \%$, respectively, 4.4, 3.2, and 1.2 times the corresponding values at the national scale. Along with this rapid urban land expansion in the MSHAs of western China, more than $90 \%$ of buildings collapsed by earthquakes in western China were found in small cities.

In light of inadequate planning for earthquake in the small cities of western China, we urge that more attention from researchers and the authorities should be paid to these cities to pursue a safer and more sustainable city development. Specifically, changing the high-density buildings located above the earthquake fault zones in the small cities of western China to other uses, such as green-belt parks, and conducting the detection of active faults in these previously neglected cities would be two promising ways to mitigate earthquake risks. The findings of our study also indicate that developing countries with rapid urban expansion and vulnerable buildings to earthquakes should plan their urban land expansion more wisely in the future.

Acknowledgements This research was supported in part by the National Natural Science Foundation of China (Grant Nos. 41621061 and 41501092), the Beijing Nova Program (Grant No. Z181100006218049), and the 111 Project "Hazard and Risk Science Base at Beijing Normal University" under Grant No. B08008 (Ministry of Education and State Administration of Foreign Expert Affairs of China).

Open Access This article is distributed under the terms of the Creative Commons Attribution 4.0 International License (http://crea tivecommons.org/licenses/by/4.0/), which permits unrestricted use, distribution, and reproduction in any medium, provided you give appropriate credit to the original author(s) and the source, provide a link to the Creative Commons license, and indicate if changes were made.

\section{References}

AQSIQ (General Administration of Quality Supervision, Inspection, and Quarantine of P.R.C.), and SAC (Standardization Administration of the People's Republic China). 2008. The Chinese seismic intensity scale. Beijing: AQSIQ and SAC.

AQSIQ (General Administration of Quality Supervision, Inspection, and Quarantine of the People's Republic of China). 2015. Seismic ground motion parameter zonation map of China GB18306-2015. Beijing: China Standards Publishing House.

Bai, X., P. Shi, and Y. Liu. 2014. Society: Realizing China's urban dream. Nature News 509(7499): 158.
Bilham, R. 1988. Earthquakes and urban growth. Nature 336(6200): 625.

Bilham, R. 2009. The seismic future of cities. Bulletin of Earthquake Engineering 7(4): 839-887.

Chatterjee, S., A. Hadi, and B. Price. 2000. Regression analysis by example. New York: Wiley.

Chen, Z., B. Yu, Y. Hu, C. Huang, K. Shi, and J. Wu. 2015. Estimating house vacancy rate in metropolitan areas using NPPVIIRS nighttime light composite data. IEEE Journal of Selected Topics in Applied Earth Observations and Remote Sensing 8(5): 2188-2197.

Dou, Y., Q. Huang, C. He, S. Meng, and Q. Zhang. 2018. Rapid population growth throughout Asia's earthquake-prone areas: A multiscale analysis. International Journal of Environmental Research and Public Health 15(9): 1893.

Dou, Y., Z. Liu, C. He, and H. Yue. 2017. Urban land extraction using VIIRS nighttime light data: An evaluation of three popular methods. Remote Sensing 9(2): 175.

Du, S., C. He, Q. Huang, and P. Shi. 2018. How did the urban land in floodplains distribute and expand in China from 1992-2015? Environmental Research Letters 13(3): Article 034018.

Ehrlich, D., M. Melchiorri, A.J. Florczyk, M. Pesaresi, T. Kemper, C. Corban, F.S.M. Carneiro, M. Schiavina, et al. 2018. Remote sensing derived built-up area and population density to quantify global exposure to five natural hazards over time. Remote Sensing 10(9): 1378. https://doi.org/10.3390/rs10091

Ehrlich, D., and P. Tenerelli. 2013. Optical satellite imagery for quantifying spatio-temporal dimension of physical exposure in disaster risk assessments. Natural Hazards 68(3): 1271-1289.

Gao, M. 2003. Characteristics of the earthquake disaster and countermeasures of mitigation in the metropolitan. Earthquake Research in China 19(2):103-108 (in Chinese).

Gao, B., Q. Huang, C. He, Z. Sun, and D. Zhang. 2016. How does sprawl differ across cities in China? A multi-scale investigation using nighttime light and census data. Landscape and Urban Planning 148: 89-98.

Giardini, D., G. Grunthal, K.M. Shedlock, and P. Zhang. 1999. The GSHAP global seismic hazard map. Annali Di Geofisica 42(6): $1225-1230$.

Granger, C.W.J. 1969. Investigating causal relations by econometric models and cross-spectral methods. Econometrica: Journal of the Econometric Society 37(3): 424-438.

He, C., Q. Huang, Y. Dou, W. Tu, and J. Liu. 2016. The population in China's earthquake-prone areas has increased by over 32 million along with rapid urbanization. Environmental Research Letters 11(7): Article 074028.

Holzer, T.L., and J.C. Savage. 2013. Global earthquake fatalities and population. Earthquake Spectra 29(1): 155-175.

Jiang, X., J. Song, Y. Lin, and Y. Gong. 2018. A practical approach to constructing hierarchical networks for urban hazard mitigation planning using GIS: The case of Futian, Shenzhen. International Journal of Disaster Risk Reduction 28: 629-639.

Li, G., S. Sun, and C. Fang. 2018. The varying driving forces of urban expansion in China: Insights from a spatial-temporal analysis. Landscape and Urban Planning 174: 63-77.

Liu, X. 2015. The main changes of the Seismic Ground Motion Parameter Zonation Map of China (GB18306-2015). China Standards Review 2015(09): 23-26 (in Chinese).

Liu, Z., C. He, and J. Wu. 2016. General spatiotemporal patterns of urbanization: An examination of 16 World cities. Sustainability 8(1): 41

Liu, X., X. Li, Y. Chen, Z. Tan, S. Li, and B. Ai. 2010. A new landscape index for quantifying urban expansion using multitemporal remotely sensed data. Landscape Ecology 25(5): 671-682. 
Liu, J., J. Zhan, and X. Deng. 2005. Spatio-temporal patterns and driving forces of urban land expansion in China during the economic reform era. AMBIO: A Journal of the Human Environment 34(6): 450-455.

López, E., G. Bocco, M. Mendoza, and E. Duhau. 2001. Predicting land-cover and land-use change in the urban fringe: A case in Morelia city, Mexico. Landscape and Urban Planning 55(4): 271-285.

Ma, Q., C. He, and J. Wu. 2016. Behind the rapid expansion of urban impervious surfaces in China: Major influencing factors revealed by a hierarchical multiscale analysis. Land Use Policy 59: 434-445.

Martins, V, P. Cabral, and D. Sousa. 2012. Urban modelling for seismic prone areas: The case study of Vila Franca do Campo (Azores Archipelago, Portugal). Natural Hazards and Earth System Sciences 12(9): 2731-2741.

McGarigal, K. 2014. FRAGSTATS help. Documentation for FRAGSTATS, 4. https://www.umass.edu/landeco/research/fragstats/ documents/fragstats.help.4.2.pdf. Accessed 6 Dec 2018.

MOHURD (Ministry of Housing and Urban-Rural Development of China). 2007. Standard for urban planning on earthquake resistance and disaster prevention. http://www.mohurd.gov.cn/ wjfb/200704/t20070427_156037.html. Accessed 6 Dec 2018 (in Chinese).

MOHURD (Ministry of Housing and Urban-Rural Development of China). 2017. The 13th five-year plan on earthquake resistance and disaster prevention in urban and rural area. http://www. mohurd.gov.cn/wjfb/201611/t20161130_229689.html. Accessed 6 Dec 2018 (in Chinese)

NBSC (National Bureau of Statistics of China). 1993. China statistical yearbook 1993. Beijing: China Statistics Press.

NBSC (National Bureau of Statistics of China). 2016. China statistical yearbook 2016. Beijing: China Statistics Press.

NBSC-PCO (State Council and the National Bureau of Statistics of China-Population Census Office). 2012. Tabulation on the 2010 population census of the People's Republic of China. Beijing: China Statistics Press.

NGDC-WDS (National Geophysical Data Center-World Data Service). 2017. Significant earthquake database. Boulder, CO: NOAA National Centers for Environmental Information (NCEI).

Potere, D., A. Schneider, S. Angel, and D.L. Civco. 2009. Mapping urban areas on a global scale: Which of the eight maps now available is more accurate? International Journal of Remote Sensing 30(24): 6531-6558.

Schneider, A., C. Chang, and K. Paulsen. 2015. The changing spatial form of cities in Western China. Landscape and Urban Planning 135: 40-61.

Schneider, A., K.C. Seto, D.R. Webster. 2005. Urban growth in Chengdu, Western China: Application of remote sensing to assess planning and policy outcomes. Environment and Planning B: Planning and Design 32(3): 323-345.

Seto, K.C., M. Fragkias, B. Güneralp, and M.K. Reilly. 2011. A metaanalysis of global urban land expansion. PloS One 6(8): Article e23777.

Shi, P. 2016. Natural disasters in China. Berlin: Springer.
Shi, Z., X. Cao, and X. Yan. 2002. Research on the earthquake occurrence in the cities of China. Earthquake Research in China 18(4): 365-370 (in Chinese).

Spence, R. 2007. Saving lives in earthquakes: Successes and failures in seismic protection since 1960. Bulletin of Earthquake Engineering 5(2): 139-251.

Storchak, D.A., D. Di Giacomo, E.R. Engdahl, J. Harris, I. Bondár, W.H.K. Lee, and A. Villaseñor. 2015. The ISC-GEM global instrumental earthquake catalogue (1900-2009): Introduction. Physics of the Earth and Planetary Interiors 239: 48-63.

Sun, B., and G. Zhang. 2018. Study on vulnerability matrices of masonry buildings of mainland China. Earthquake Engineering and Engineering Vibration 17(2): 251-259.

Tao, S., J. Fang, X. Zhao, S. Zhao, H. Shen, H. Hu, Z. Tang, Z. Wang, and Q. Guo. 2015. Rapid loss of lakes on the Mongolian Plateau. Proceedings of the National Academy of Sciences 112(7): 2281-2286.

Uysal, C., D. Maktav, and C. Small. 2018. Mapping urban growth and its relation to seismic hazards in Istanbul. Journal of the Indian Society of Remote Sensing 46(8): 1307-1322.

Wang, Z. 2008. A preliminary report on the Great Wenchuan Earthquake. Earthquake Engineering and Engineering Vibration 7(2): 225-234.

Wang, B., F. Miao, D. Lai, M. Yan, and D. Sun. 2007. The impact on the expansion of Tangshan City brought by geological disasters. Urban Problems 2007(11): 40-43 (in Chinese).

Wieland, M., M. Pittore, S. Parolai, and J. Zschau. 2012. Exposure estimation from multi-resolution optical satellite imagery for seismic risk assessment. ISPRS International Journal of GeoInformation 1(1): 69-88.

Wu, J., A. Buyantuyev, G. Jenerette, J. Litteral, K. Neil, and W. Shen. 2011. Quantifying spatiotemporal patterns and ecological effects of urbanization: A multiscale landscape approach. In Applied urban ecology: A global framework, ed. M. Richter, and U. Weiland, 33-53. Oxford: Blackwell Publishing.

Wu, Z., T. Ma, H. Jiang, and C. Jiang. 2013. Multi-scale seismic hazard and risk in the China mainland with implication for the preparedness, mitigation, and management of earthquake disasters: An overview. International Journal of Disaster Risk Reduction 4: 21-33.

Wu, J., C. Wang, X. He, X. Wang, and N. Li. 2017. Spatiotemporal changes in both asset value and GDP associated with seismic exposure in China in the context of rapid economic growth from 1990 to 2010. Environmental Research Letters 12(3): Article 034002.

$\mathrm{Xu}$, M., C. He, Z. Liu, and Y. Dou. 2016a. How did urban land expand in China between 1992 and 2015? A multi-scale landscape analysis. PloS One 11(5): Article e0154839.

Xu, W., J. Liu, G. Xu, Y. Wang, L. Liu, and P. Shi. 2016b. Earthquake disasters in China. In Natural disasters in China, ed. P. Shi, 37-72. Berlin: Springer.

Zhang, Q., Q. Lu, D. Zhong, and X. Ye. 2018. The pattern of policy change on disaster management in China: A bibliometric analysis of policy documents, 1949-2016. International Journal of Disaster Risk Science 9(1): 55-73. 